\title{
A Modified TreePM Code
}

\author{
Nishikanta Khandai and J. S. Bagla \\ Harish-Chandra Research Institute, Chhatnag Road, Jhusi, Allahabad 211019, India. \\ e-mail: nishi@hri.res.in,jasjeet@hri.res.in
}

\begin{abstract}
We discuss the performance characteristics of using the modification of the tree code suggested by Barnes (Barnes, 1990) in the context of the TreePM code. The optimisation involves identifying groups of particles and using only one tree walk to compute force for all the particles in the group. This modification has been in use in our implementation of the TreePM code for some time, and has also been used by others in codes that make use of tree structures. In this paper, we present the first detailed study of the performance characteristics of this optimisation. We show that the modification, if tuned properly can speed up the TreePM code by a significant amount. We also combine this modification with the use of individual time steps and indicate how to combine these two schemes in an optimal fashion. We find that the combination is at least a factor of two faster than the modified TreePM without individual time steps. Overall performance is often faster by a larger factor, as the scheme of groups optimises use of cache for large simulations.
\end{abstract}

Key words: Cosmology; Gravitation; Methods: numerical; Large-scale structure of universe

\section{INTRODUCTION}

Large scale structures traced by galaxies are believed to have formed by amplification of small perturbations (Peebles, 1980; Peacock, 1999; Padmanabhan, 2002; Bernardeau et al., 2002). Galaxies are highly overdense systems, matter density $\rho$ in galaxies is thousands of times larger than the average density $\bar{\rho}$ in the universe. Typical density contrast $(\delta \equiv \rho / \bar{\rho}-1)$ in matter at these scales in the early universe was much smaller than unity. Thus the problem of galaxy formation and the large scale distribution of galaxies requires an understanding of the evolution of density perturbations from small initial values to the large values we encounter today.

Initial density perturbations were present at all scales that have been observed (Spergel et al., 2007; Percival et al., 2007). The equations that describe the evolution of density perturbations in an expanding universe have been known for several decades (Peebles, 1974) and these are easy to solve when the amplitude of perturbations is small. Once density contrast at relevant scales becomes comparable to unity, perturbations becomes non-linear and coupling with perturbations at other scales cannot be ignored. The equation for evolution of density perturbations cannot be solved for generic initial conditions in this regime. N-Body simulations (e.g., see (Efstathiou et al., 1985; Bertschinger, 1998; Bagla \& Padmanabhan, 1997; Bagla, 2005)) are often used to study the evolution in this regime. Alternative approaches can be used if one requires only a limited amount of information and in such a case either quasi-linear approximation schemes (Bernardeau et al., 2002), (Zel'Dovich, 1970; Gurbatov, Saichev, \& Shandarin, 1989; Matarrese et al., 1992; Brainerd, Scherrer, \& Villumsen, 1993; Bagla \& Padmanabhan, 1994; Sahni \& Coles, 1995; Hui \& Bertschinger, 1996) or scaling relations (Davis \& Peebles, 1977; Hamilton et al., 1991; Jain, Mo, \& White, 1995; Kanekar, 2000; Ma, 1998; Nityananda \& Padmanabhan, 1994; Padmanabhan et al., 1996; Peacock \& Dodds, 1994; Padmanabhan, 1996; Peacock \& Dodds, 1996; Smith et al., 2003) suffice. However, even the approximation schemes and scaling relations must be compared with simulations before these can be used with confidence. 
Last three decades have seen a rapid development of techniques and computing power for cosmological simulations and the results of these simulations have provided valuable insight into the study of structure formation. The state of the art simulations used less than $10^{5}$ particles two decades ago (Efstathiou et al., 1988) and if the improvement had been due only to computing power then the largest simulation possible today should have been around $10^{9}$ particles, whereas the largest simulations done till date used more than $10^{10}$ particles (Springel et al., 2005). Evidently, development of new methods and optimisations has also played a significant role in the evolution of simulation studies (Efstathiou et al., 1985), (Barnes \& Hut, 1986; Greengard \& Rokhlin, 1987; Bouchet \& Hernquist, 1988; Jernigan \& Porter, 1989; Hernquist, 1990; Makino, 1990, 1991; Hernquist, Bouchet. \& Suto, 1991; Couchman, 1991; Ebisuzaki et al., 1993; Theuns, 1994; Brieu. Summers, \& Ostriker, 1995; Suisalu \& Saar, 1995; Xu, 1995; Dubinski, 1996; Kravtsov, Klypin, \& Khokhlov, 1997; Macfarland et al., 1998; Bode, Ostriker, \& Xu, 2000; Brieu \& Evrard, 2000; Dehnen, 2000; Knebe, Green, \& Binney, 2001; Springel, Yoshida, \& White, 2001; Kawai \& Makino, 2001; Makino, 2002; Dehnen, 2002; Bagla, 2002; Bagla \& Rav, 2003; Makino et al., 2003; Bode \& Ostriker, 2003; Rav \& Bagla, 2004; Dubinski et al., 2004; Makino, 2004; Springel, 2005; Merz, Pen, \& Trac, 2005; YYoshikawa \& Fukushige, 2005; Wadsley, Stadel, \& Quinn, 2004; Thacker \& Couchman, 2006) Along the way, code developers have also successfully met the challange posed by the emergence of distributed parallel programming.

In this paper, we discuss the performance characteristics of an optimisation for tree codes suggested by Barnes (Barnes, 1990). We do this in the context of the TreePM method (Bagla, 2002; Bagla \& Ray, 2003) where the tree method is used for computing the short range force. The TreePM method brings in an additional scale into the problem, i.e., the scale upto which the short range force is computed and this leads to non-trivial variations in error in force.

The paper is organised as follows: we introduce the TreePM method in $\S 2$, and discuss the optimisation scheme in $\S 3$. Performance of the optimisation scheme is discussed in $\S 4$, and we discuss combining this with individual time steps for particles in $\S 5$. We end with a discussion in $\S 6$.

\section{THE TREEPM ALGORITHM}

The TreePM algorithm (Bagla, 2002; Bagla \& Rav, 2003) is a hybrid N-Body method which combines the BH-Tree method (Barnes \& Hut, 1986) with the PM method (Bagla \& Padmanabhan, 1997; Merz, Pen. \& Trac, 2005), (Klvpin \& Shandarin, 1983; Miller, 1983; Bouchet \& Kandrup, 1985; Bouchet, Adam, \& Pellat, 1985; Hockney \& Eastwood, 1988). The TreePM method explicitly breaks the potential into a short-range and a longe-range component at a scale $r_{s}$. The PM method is used to calculate long-range force and the short-range force is computed using the BH Tree method. Use of the BH Tree for short-range force calculation enhances the force resolution as compared to the PM method.

The gravitational force is divided into a long range and a short range part using partitioning of unity in the Poisson equation.

$$
\begin{aligned}
\phi_{k} & =-\frac{4 \pi G \rho_{k}}{k^{2}} \\
& =-\frac{4 \pi G \rho_{k}}{k^{2}} \exp \left(-k^{2} r_{s}^{2}\right)-\frac{4 \pi G \rho_{k}}{k^{2}}\left[1-\exp \left(-k^{2} r_{s}^{2}\right)\right] \\
& =\phi_{k}^{l r}+\phi_{k}^{s r} \\
\phi_{k}^{l r} & =-\frac{4 \pi G \rho_{k}}{k^{2}} \exp \left(-k^{2} r_{s}^{2}\right) \\
\phi_{k}^{s r} & =-\frac{4 \pi G \rho_{k}}{k^{2}}\left[1-\exp \left(-k^{2} r_{s}^{2}\right)\right]
\end{aligned}
$$

Here $\phi_{k}^{s r}$ and $\phi_{k}^{l r}$ are the short-range and long-range potentials in Fourier space. $\rho$ is the density, G is the gravitational coupling constant and $r_{s}$ is the scale at which the splitting of the potential is done. The longrange force is solved in Fourier space with the PM method and the short-range force is solved in real space with the Tree method. The short range force in real space is:

$$
\mathbf{f}^{s r}(\mathbf{r})=-\frac{G M \mathbf{r}}{r^{3}}\left[\operatorname{erfc}\left(\frac{r}{2 r_{s}}\right)+\frac{r}{r_{s} \sqrt{\pi}} \exp \left(-\frac{r^{2}}{4 r_{s}^{2}}\right)\right]
$$


Here erfc is the complementary error function.

The short range force is below $1 \%$ of the total force at $r \geq 5 r_{s}$. The short range force is therefore computed within a sphere of radius $r_{c u t} \simeq 5 r_{s}$. The short range force is computed using the $\mathrm{BH}$ tree method. The tree structure is built out of cells and particles. Cells may contain smaller cells (subcells) within them. Subcells can have even smaller cells within them, or they can contain a particle. In three dimensions, each cubic cell is divided into eight cubic subcells. Cells, as structures, have attributes like total mass, location of centre of mass and pointers to subcells. Particles, on the other hand have the usual attributes: position, velocity and mass.

Force on a particle is computed by adding contribution of other particles or of cells. A cell that is sufficiently far away can be considered as a single entity and we can add the force due to the total mass contained in the cell from its centre of mass. If the cell is not sufficiently far away then we must consider its constituents, subcells and particles. Whether a cell can be accepted as a single entity for force calculation is decided by the cell acceptance criterion (CAC). We compute the ratio of the size of the cell $d$ and the distance $r$ from the particle in question to its centre of mass and compare it with a threshold value

$$
\theta=\frac{d}{r} \leq \theta_{c}
$$

The error in force increases with $\theta_{c}$. Poor choice of $\theta_{c}$ can lead to significant errors Salmon \& Warren, 1994). Many different approaches have been tried for the CAC in order to minimize error as well as CPU time usage (Salmon \& Warren, 1994; Springel. Yoshida, \& White, 2001). The tree code gains over direct summation as the number of contributions to the force is much smaller than the number of particles.

The TreePM method is characterised therefore by three parameters, $r_{s}, r_{c u t}$ and $\theta_{c}$. For a discussion on the optimum choice of these parameters the reader is referred to (Bagla \& Ray, 2003).

\section{THE SCHEME OF GROUPS}

We first describe an optimization scheme due to (Barnes, 1990), given in the paper with a curious title A modified tree code. Don't laugh, it runs. This scheme is easily portable to any N-body algorithm that uses tree data structures to compute forces. The origin of the optimisation is in the realisation that the tree walk used for computing forces is computationally the most expensive component of a tree code. The idea is to have a common interaction list for a group of particles that is sufficiently small. Given that we are working with a tree code, it is natural to identify a cell in the tree structure as a group. One can then add the contribution of particles within the group using direct pair summation. The cell acceptance criterion $(\mathrm{CAC})$ for the tree walk needs to be modified in order to take the finite size of the group into account. In our implementation of the TreePM method, we modified the standard CAC in the following manner:

$$
d \leq\left(r-r_{m}\right) \theta_{c}
$$

where $r_{m}$ is the distance between the centre of mass of the group, and the group member that is farthest from the centre of mass. This is calculated once before the force calculation and does not add much in terms of overhead.

The modified CAC can be thought of as the standard CAC with a distance dependent $\theta_{c}$, with the value of $\theta_{c}$ decreasing at small $r$. As we require a larger number of operations for smaller $\theta_{c}$, each tree walk with the modified CAC is expected to require more CPU time than a tree walk with the standard CAC. However, as we do a tree walk for a group of particles in one go, CPU time is saved as the time taken for tree walk per particle comes down.

There is an overhead as there is a pair-wise force calculation within the group. The cost of this overhead increases as the square of the number of particles in the group. In order to keep the overhead small, one would like the group to be sufficiently small compared to the size of the N-Body simulation and hence a maximum size $c_{\max }$ and an upper bound on the number of particles in the group $n_{p_{\max }}$ is used. An upper limit on the size of the group is pertinent because of the indirect effect through the change in the CAC. The effect of the additional parameter $c_{\max }$ with the modified CAC will be seen when we discuss errors in section 4. Our implementation of the modified method by using a different definition of groups, with the additional parameter $c_{\max }$ and the modified CAC (eq.5) ensure that the short-range force is extremely accurate. 
This is different from previous implementations (Barnes, 1990; Makino, 1991; Yoshikawa \& Fukushige, 2005; Wadsley, Stadel, \& Quinn, 2004) where the group scheme was parametrized by just one parameter $n_{p_{\max }}$ and the standard CAC (eq.4) used for tree traversal. We note in passing that the modified CAC is crucial in order to limit errors. Indeed, we find that working with the standard CAC leads to errors in short range force that are orders of magnitude larger.

\subsection{Estimating Speedup}

We model the modified Tree/TreePM method with the aim of estimating the speedup that can be achieved. If $N$ is the total number of particles, $n_{p}$ the typical number of particles in a group and $n_{g}$ the number of groups then clearly we expect $n_{g} \times n_{p}=N$. The total time required for force calculation is a sum of the time taken up by the tree walk and the time taken up by pair wise calculation within the group. Actual calculation of the force, once the interaction list has been prepared takes very little time and can be ignored in this estimate, as can the time taken to construct the tree structure. The time taken is:

$$
T_{g}=\alpha n_{g} \ln N+\beta n_{g} n_{p}^{2}=\alpha \frac{N}{n_{p}} \ln N+\beta N n_{p}
$$

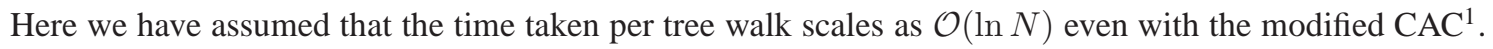
The time taken is smallest when

$$
n_{p}=\left(\frac{\alpha \ln N}{\beta}\right)^{1 / 2} \quad ; \quad T_{g_{\min }}=2 \beta N n_{p}=2 \alpha \frac{N}{n_{p}} \ln N
$$

Thus the optimum number of particles in the group scales weakly with the total number of particles. In the optimum situation, we expect the tree walk and the pair wise components to take the same amount of CPU time.

For comparison, the time taken for force calculation in the standard TreePM is:

$$
T=\alpha N \ln N
$$

and we make the simplifying assumption that $\alpha$ is same for the two cases. The expected speed up is then given by:

$$
\frac{T}{T_{g_{\text {min }}}}=\frac{1}{2}\left(\frac{\alpha \ln N}{\beta}\right)^{1 / 2} \quad .
$$

The speedup for the optimum configuration scales in the same manner as the typical number of particles per group.

A more detailed analysis of this type can be found in (Makino, 1991).

The calculation we have presented above is approximate and ignores several factors, some of these have already been highlighted above. There are other subtleties like the role played by the finite range $r_{c u t}$ over which the short range force is calculated. The size of a group $\left(c_{\max }\right)$ cannot be varied continuously, and hence $n_{p}$ is also restricted to a range of values. Further, the number of operations do not translate directly into CPU time as some calculations make optimal use of the capabilities of a CPU while others do not. For example, the pair wise calculation is likely to fare better on processors with a deep pipeline for execution whereas tree walk can not exploit this feature. The finite bandwidth of the CPU-memory connection also has an impact on the scaling with $N$ for large $N$. In the following section, we discuss the implementation of the modified TreePM method and the timing of the code with different values of parameters.

\footnotetext{
1 This is an approximation as we expect the tree walk to depend on $c_{\max }, n_{p_{\max }}$ and $\theta_{c}$ as well. The finite size of groups should lead to deviations from the $\mathcal{O}(\ln N)$ variation and the deviation should scale as the ratio of the volume of the group and the volume of the simulation box. As this ratio becomes smaller for large simulation boxes, we feel that the approximation we have made is justified.
} 

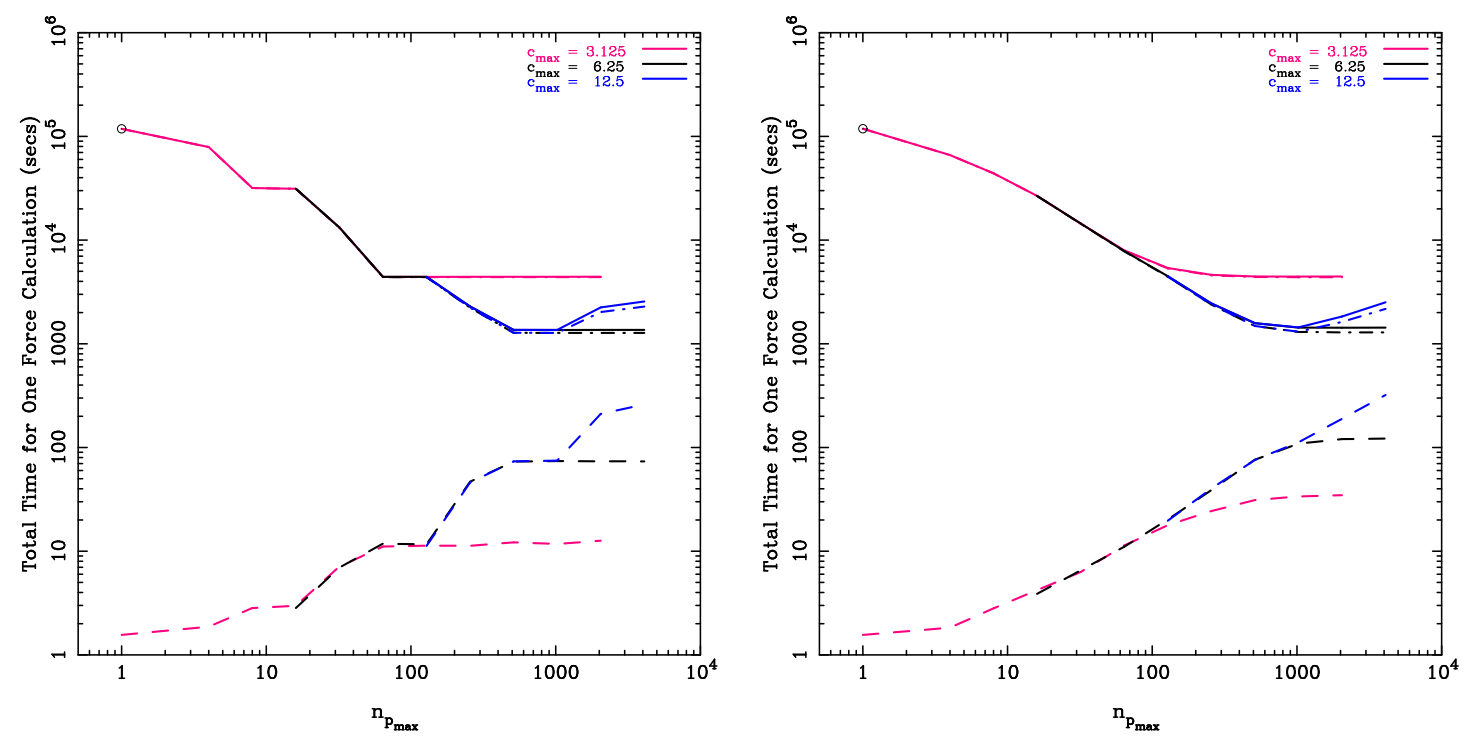

Fig. 1 Time taken for computation of the short term force in the Modified TreePM method for an unclustered (left panel) and a clustered (right panel) distribution. Solid lines represent the time taken by a complete short-range force calculation. Dashed lines are the contribution to the force due to pairs within a group, the intra-group contribution. Dot-Dashed lines are the contributions to force due to tree walk. Purple, black and blue lines are for $c_{\max }=3.125,6.25,12.5$ respectively

\section{A MODIFIED TREEPM ALGORITHM WITH THE SCHEME OF GROUPS}

Tests of the TreePM method have shown that $95-98 \%$ of the time goes into the short-range force calculation. Keeping this in mind the scheme of groups was introduced to optimize the short-range force calculation in terms of speed. A welcome feature is more accurate force computation. Since the optimum set of TreePM parameters have been discussed in (Bagla \& Ray, 2003), we now look for the optimum choice of the additional parameters, $c_{\max }$ and $n_{p_{\max }}$, which describe the Modified TreePM algorithm. The analysis that follows is divided into two parts. First we look at the optimum values of $c_{\max }$ and $n_{p_{\max }}$ which minimise the time for short range force computation. Second, we study errors in total and short range force with this new scheme.

\subsection{Optimum Parameters of The Modified TreePM Algorithm}

We choose $r_{s}=1, r_{c u t}=5.2 r_{s}$ and $\theta_{c}=0.5$ for the discussion that follows. With this choice the error in force for $99 \%$ of the particles is less than a few percent (Bagla \& Ray, 2003). We present analysis of performance of the modified TreePM for two different particle distributions taken from an $N$-body simulation, with $N=N_{b o x}^{3}=200^{3}$.

- An unclustered distribution that corresponds to the initial conditions of an $N$-body simulation.

- A clustered distribution taken again from the same $N$-body simulation. The scale of non-linearity for the clustered distribution is 8 grid lengths.

We have verified that the nature of results does not change significantly for simulations with the number of particles ranging from $32^{3}$ to $256^{3}$. 
Table 1 The following table lists the optimum values of $\left(c_{\max }, n_{p_{\max }}\right)$ for simulations of various sizes but having the same TreePM parameters: $r_{s}=1, r_{c u t}=5.2 r_{s} \theta_{c}=0.5$.

\begin{tabular}{||c|l|c||}
\hline \hline$N_{\text {box }}=N^{1 / 3}$ & $c_{\max }^{o p t}$ & $n_{p_{\max }}^{\text {opt }}$ \\
\hline \hline 64 & 4.0 & $\geq 1024$ \\
\hline 128 & 4.0 & $\geq 1024$ \\
\hline 160 & 5.0 & $\geq 1024$ \\
\hline 200 & 6.25 & $\geq 1000$ \\
\hline \hline
\end{tabular}

In figure 1 we show the time taken for computing the the short-range force (solid line) and determine the values of $\left(c_{\max }, n_{p_{\max }}\right)$ for which this timing is a minimum. Two leading contributions to the calculation of short range force are shown separately:

- Intra-group particle-particle contribution (dashed line).

- Time take for tree-walk and the related force calculation (dot-dashed line).

Given that a group is a cell with maximum width

$$
c_{\max }=\frac{N_{b o x}}{2^{m}}, \quad(m \text { is an integer }),
$$

$c_{\max }$ can take therefore only discrete values. We choose to restrict upto $c_{\max } \sim 2 r_{\text {cut }}$ as for larger cells, the dominant contribution to force on a given particle arises from the intra-group particle-particle interaction and the time taken for this is a sensitive function of the amplitude of clustering.

The time taken for computing the short range force in both, unclustered (left panel) as well as clustered (right panel), distributions is qualitatively described by our model (see eqn.(6)). The pairwise force increases linearly with $n_{p_{\max }}, n_{p_{\max }}$ is the maximum number of particles in a group and scales as $n_{p}$ where $n_{p}$ is the average number of particles in a group. The time taken for tree-walk decreases as $n_{p_{\max }}^{-0.65}$, reaches a minimum and then increases with $n_{p_{\max }}$ (blue line) for the largest $c_{\max }$ used here. For other values of $c_{\max }$ we see the timing levelling off near the minimum. The scaling as $n_{p_{\text {max }}}^{-0.65}$ is different from $1 / n_{p}$ we used in the analytical model and the reason for this is likely to be in the approximations we used. We find that the scaling approaches $1 / n_{p_{\max }}$ as we consider simulations with a larger number of particles. One crucial reason for different scaling is the modified CAC we use here. This effectively leads to a smaller $\theta_{c}$ for cells closer to the group and the number of such cells increases with $c_{\max }$.

In both cases the total time is still dominated by the tree-walk. The plateaus in the plots often indicate that the number of particles in a group of maximum size $c_{\max }$ have saturated. At initial times where the fluctuations are small there is also a lower bound on the number of particles contained in a group. In the clustered distribution there is no such lower bound but an upper bound, larger than the corresponding upper bound in case of the unclustered distribution, exists and is dictated by the amplitude of clustering in the distribution of particles.

From figure 1 we see that the optimum values of $\left(c_{\max }, n_{p_{\max }}\right)=(12.5,1024) \&(6.25, \geq 1024)$ given by the minima of the solid blue line and the plateau of the solid black line respectively. In the latter case the time taken does not change for $n_{p_{\max }} \geq 1024$ and we consider this to be a useful feature that makes the $c_{\max }=6.25$ a better choice as fine tuning of $n_{p_{\max }}$ is not required. For the optimum $\left(c_{\max }, n_{p_{\max }}\right)$ one can see that force computation takes the same time for the clustered and the unclustered distributions. Table 1 lists optimum values for $\left(c_{\max }, n_{p_{\max }}\right)$ for N-Body simulations with different numbers of particles. These numbers indicate that a good choice for $c_{\max }$ is one which is closest to $r_{\text {cut }}$, i.e. $c_{\max } \sim r_{\text {cut }}$. The parameter $n_{p_{\max }}$ can be taken to be $10^{3} \leq n_{p_{\max }}$ as we find little variation beyond this point.

One can get an estimate of the overheads for the group scheme by looking at the limit of $n_{p_{\max }} \rightarrow 1$. Here we compare the performance of the TreePM with the modified code by plotting the time taken by the former as a large dot on the same panel where the time taken for the modified code is shown in the form of curves. The difference between these timings is around $0.1 \%$. 


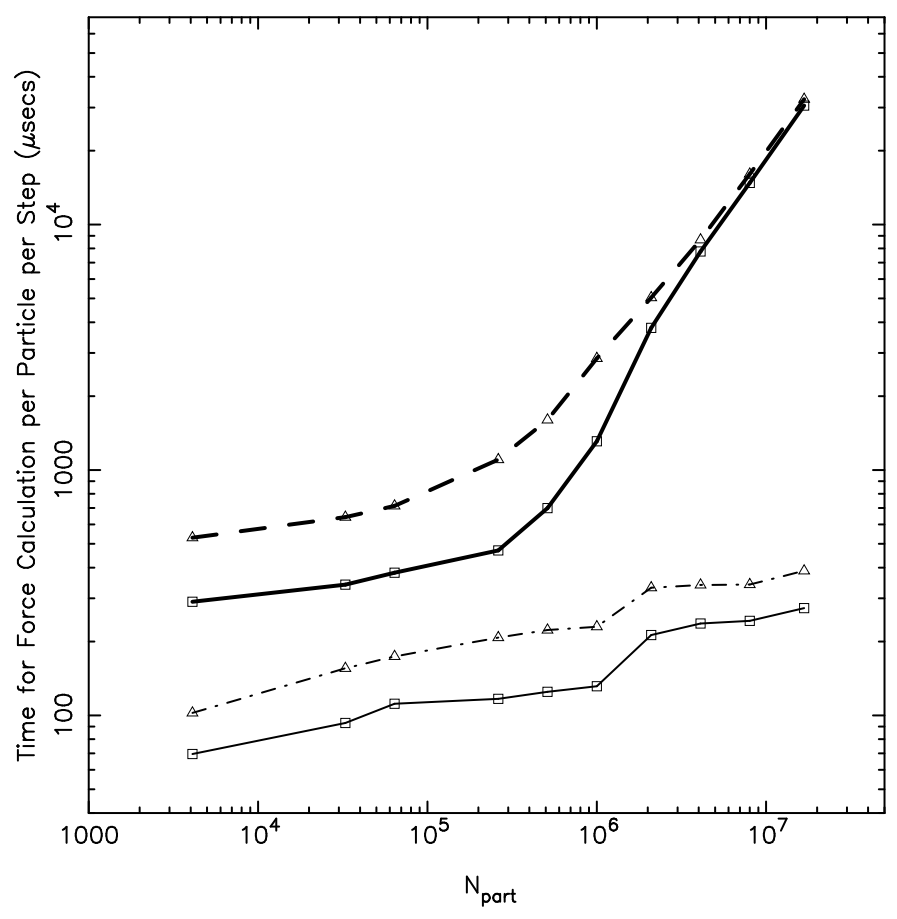

Fig. 2 Time taken for short-range force calculation per particle per step for $N=32^{3}$ to $N=$ $256^{3}$ for the TreePM (thick line) and the Modified TreePM (thin line). The solid line shows the performance of the codes on a single core if Intel $5160(3.0 \mathrm{GHz})$ processor and the dashed line shows the performance on a single core of the AMD Barcelona $(2.1 \mathrm{GHz})$ processor.

The speedup for the optimal configuration of the modified TreePM, as compared with the base TreePM code is $\sim 83$. This is a huge gain and has to do with better utilisation of the CPU cache. The speedup is less impressive for smaller simulations, and is larger for bigger simulations. This is shown in figure 2 where we plot the time taken for force calculation per particle per step as a function of the total number of particles in the simulation. This is shown for the TreePM as well as the modified TreePM codes. Performance on two different types of processors is shown here to demonstrate that the optimisation works equally well on these. One can see that the TreePM code becomes (CPU-Memory) bandwidth limited for simulations with more than $64^{3}$ particles and the time taken increases more rapidly than $\mathcal{O}(\ln N)$. This does not happen in case of the modified TreePM where the scaling is $\mathcal{O}(\ln N)$ throughout. It is this difference that leads to impressive speedup for large simulations. For simulations with up to $64^{3}$ particles we get a speedup by a factor of four.

\subsection{Errors in the Modified TreePM Force}

We now study errors in force for the modified TreePM force. Errors are calculated with respect to a reference force computed with very conservative values of TreePM parameters: $\theta_{c}=0.01, r_{s}=4.0, r_{c u t}=5.2 r_{s}$. With these values the reference force is accurate to $0.1 \%$ (Bagla \& Ray, 2003).

$$
\epsilon=\frac{\left|\mathbf{f}_{\text {ref }}-\mathbf{f}\right|}{\left|\mathbf{f}_{\text {ref }}\right|}
$$

Here $\epsilon, \mathbf{f}_{\text {ref }}, \mathbf{f}$ are the relative error, reference force and the typical force in a simulation. We calculate errors for two distributions of particles: 

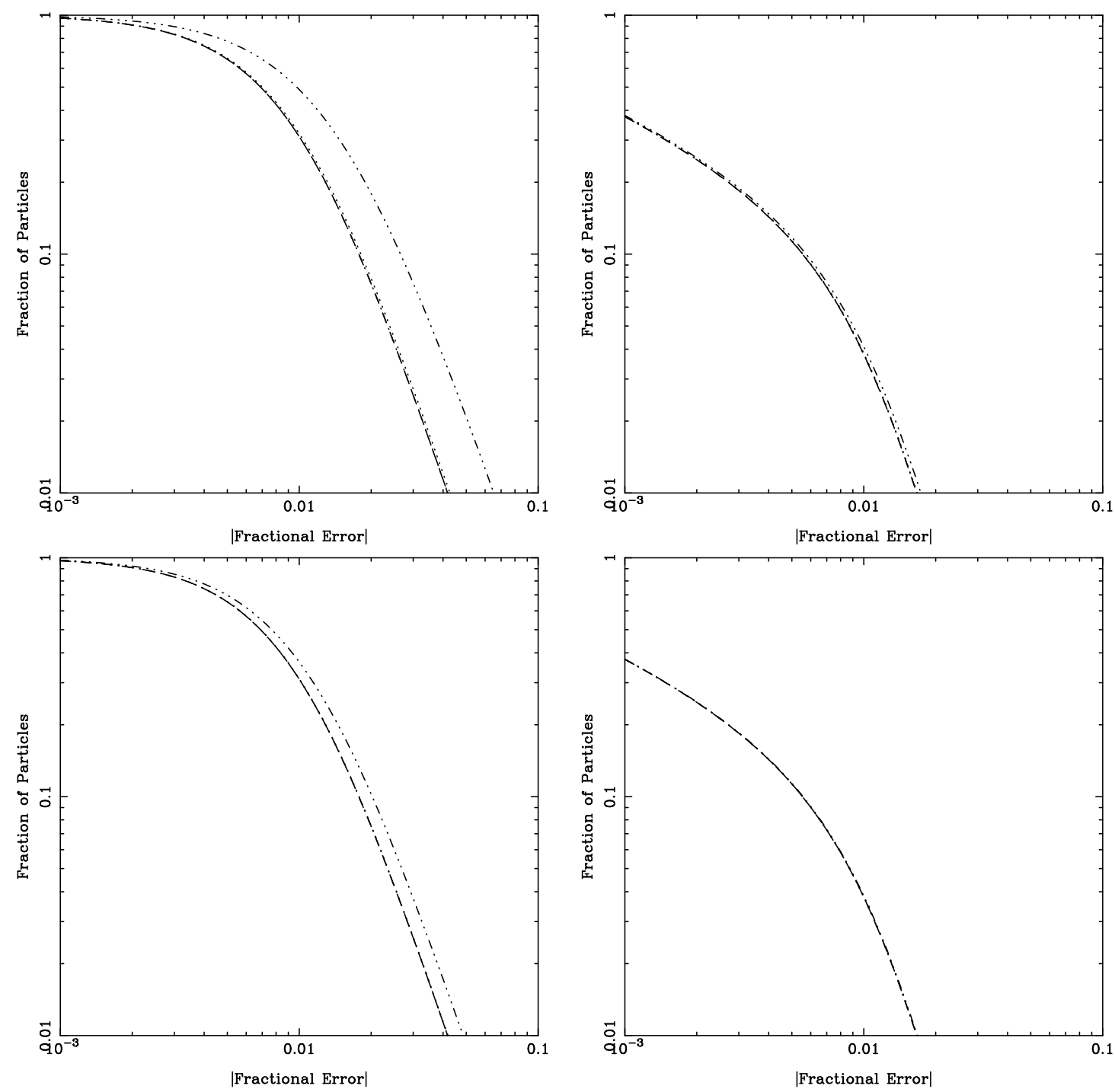

Fig. 3 Distribution of errors in total force for different values of $\theta_{c}$ with $c_{\max }=2.0$ for unclustered (top left panel) and clustered (top right panel) distributions. Dashed, dot-dash-dotdash, dotted, dash-dot-dot-dot lines are for $\theta_{c}=0.1,0.2,0.3$ and 0.5 respectively. We used $r_{s}=1.0, r_{c u t}=5.2 r_{s}$ for these plots. The corresponding plots for $c_{\max }=4.0$ are shown in lower left and lower right panels.

- A uniform (unclustered) distribution.

- A clustered distribution taken from an $N$-body simulation.

Both distributions have $N_{b o x}^{3}=N=128^{3}$ particles. The exercise we follow is similar to (Bagla \& Ray, 2003) but now we wish to highlight the effect of groups on errors in force.

Figure 3 shows the distribution of errors for different values of $\theta_{c}$. The results are shown for both the distributions being studied here: the unclustered distribution (left panels) and the clustered distribution (right panels). The top row is with $c_{\max }=2.0$ and the lower row is for $c_{\max }=4.0$. We used $r_{s}=1.0$ and 

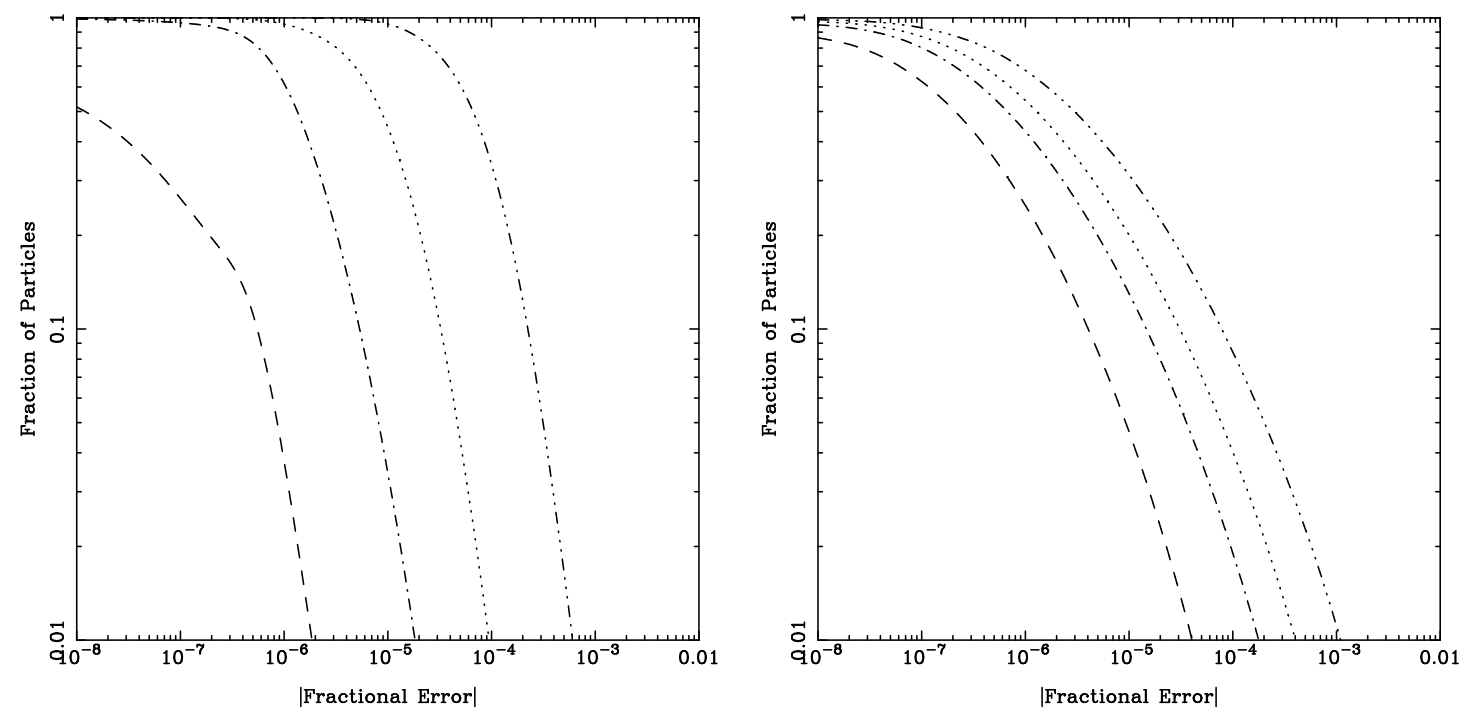

Fig. 4 Distribution of errors in short range force for different values of $\theta_{c}$ for unclustered (left panel) and clustered (right panel) distributions. Dashed, dot-dash-dot-dash, dotted, dash-dot-dotdot lines are for $\theta_{c}=0.1,0.2,0.3$ and 0.5 respectively. $c_{\max }=4.0, r_{s}=1.0, r_{c u t}=5.2 r_{s}$ was used for these plots.

$r_{c u t}=5.2 r_{s}$ for this figure. In the case of the unclustered distribution error decreases with $\theta_{c}$ but saturates at $\theta_{c}=0.3$ and does not decrease as $\theta_{c}$ is decreased further. The situation is different for the clustered distribution where the errors are not sensitive to $\theta_{c}$. This suggests that the errors are dominated by the longrange force. The unclustered distribution has larger errors than the clustered distribution. This is because the net force on each particle in the unclustered distribution is small, whereas force due to a cell with many particles is large and many such large contributions have to cancel out to give a small net force. Numerical errors in adding and subtracting these large numbers seem to systematically give a net large error. Larger cells contribute for larger $\theta_{c}$ hence the variation with $\theta_{c}$ is more dramatic in the unclustered case. This effect is apparent in the discussion of the short-range force. With $\theta_{c}=0.3,1 \%$ of particles have errors in total force greater than $4 \%$ in the unclustered case and $1.6 \%$ in clustered case.

The effect of the modified CAC (eqn. (5)) is seen by comparing the plots of figure 3 for the unclustered distribution. The modified CAC is more stringent for larger value of $c_{\max }$ and this is clearly seen in the error for $\theta_{c}=0.5$. There is a lack of variation in errors with $\theta_{c}$ for $\theta_{c}<0.5$ indicating that at this stage the dominant contribution to errors is from the long range force calculation. The short-range force is more accurate with a larger $c_{\max }$ due to two reasons:

- The modified CAC has an $r$ dependent opening angle threshold and requires a smaller $\theta_{c}$ at small distances. This is likely to reduce errors.

- The number of particles in a group is larger for larger $c_{\max }$. As the contribution of force from these particles is computed by a direct summation over pairs, the errors are negligible.

One may raise the concern that the errors in the present approach are likely to depend on location of a particle within the group. We have checked for anisotropies in error in force calculation in groups that may result and we do not find any noteworthy anisotropic component.

Next we look at the errors in short-range force for the same distributions (unclustered and clustered) of particles for various values of $\theta_{c}$. The reference short-range force was computed with $\theta_{c}=0.01, r_{s}=1.0$, $r_{c u t}=5.2 r_{s}$ and $c_{\max }=4.0$. We only varied $\theta_{c}$ and continued to use $r_{s}=1.0, r_{c u t}=5.2 r_{s}, c_{\max }=4.0$ 

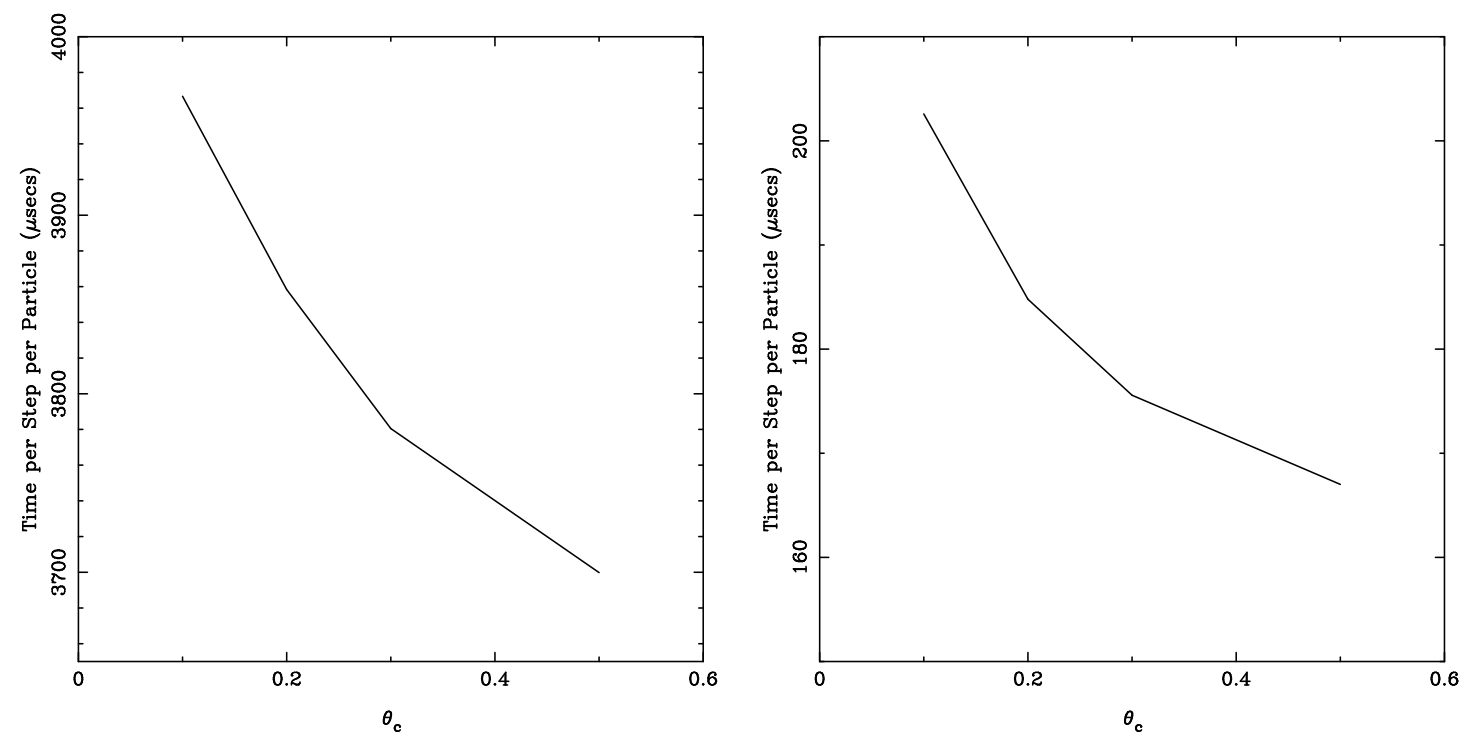

Fig. 5 Scaling of the time taken for short-range force calculation with $\theta_{c}$ for the TreePM (left panel) and the modified TreePM (right panel).

for computing the short-range force and then the errors. For the purpose of computing errors in the short range force, we cannot vary $r_{s}$ between the reference and the test model.

The effect of decreasing $\theta_{c}$ is more dramatic on errors in the short-range force. For the unclustered case the errors for $1 \%$ of the particles decreases by nearly 2.5 decades to $2 \times 10^{-4 \%}$ for $\theta_{c}=0.1$. In the clustered case the errors for $1 \%$ of the particles decrease by nearly 1.5 decades to $3.4 \times 10^{-2} \%$ for $\theta_{c}=0.1$. One can obtain very high accuracy in short-range force by taking $\theta_{c}=0.2$. As the short range force is the dominant one at small scales, the TreePM code can be used to follow the local dynamics fairly well by using a smaller $\theta_{c}$. The impact of a small $\theta_{c}$ on CPU time remains to be seen though.

In figure 5 we look at how the CPU time for force calculation scales with $\theta_{c}$ for the TreePM (left panel) and the modified TreePM (right panel). We compute the time taken for short-range force calculation per particle per timestep. We have seen in figure 1 and the corresponding discussion that clustering does not seriously affect the performance of the TreePM code. We therefore do not repeat the exercice for distributions with different levels of clustering. We performed the short-range force timing on a clustered distribution taken from an $N$-body simulation with $N_{b o x}^{3}=N=128^{3}$. We used $r_{s}=1.0$ and $r_{c u t}=4.0$ for the TreePM and the modified TreePM. In addition $c_{\max }=4.0, n_{p_{\max }}=1024$ were used for timing the modified TreePM. When $\theta_{c}$ is decreased from 0.5 to 0.2 the time for force computation per particle increases by $7.2 \%$ for the TreePM and $21 \%$ for the modified TreePM. The speedup of the modified TreePM over the TreePM when $\theta_{c}$ is reduced from 0.5 to 0.2 decreases from 22.2 to 19.6 , respectively. A nice feature of TreePM codes is that unlike tree codes, the CPU time taken by TreePM codes is less sensitive to $\theta$ 2. Thus one can obtain much higher accuracy for the short range force with a TreePM code for a considerably smaller cost in terms of the CPU time.

\section{A HIERARCHY OF TIMESTEPS}

Due to the existence of a large range of dynamical time scales in a simulation of large scale structures, computing forces for slowly moving particles at every timestep is not required. It is better to integrate

\footnotetext{
${ }^{2}$ For example, the variation in CPU time for a tree code increases by about $500 \%$ for the same change in $\theta_{c}$ for a simulation with $N \approx 10^{4}$, and the increase in CPU time is larger for simulations with a larger number of particles (Hernquist, 1987 ).
} 
Table 2 This table lists the time taken for a complete simulation run for the unoptimised TreePM, TreePM with hierarcical time steps, TreePM with the group scheme, and finally the TreePM with the group scheme as well as the hierarchical time steps and their speedup with respect to the base TreePM.

\begin{tabular}{||c|c|c|c|c||}
\hline \hline Run & Groups & Individual Timesteps & Time (secs) & Speedup w.r.t Run 1 \\
\hline \hline 1 & No & No & 401983 & 1.0 \\
\hline 2 & No & Yes & 145240 & 2.77 \\
\hline 3 & Yes & No & 67639 & 5.94 \\
\hline 4 & Yes & Yes & 31612 & 12.72 \\
\hline \hline
\end{tabular}

the orbits of rapidly moving particles with a smaller timestep than those that move relatively slowly, this reduces the number of force calculations that are required. As force calculation is the most time consuming component of an N-Body code, this results in a significant reduction of the CPU time required. We have implemented a hierarchical time integrator similar to that used in GADGET-2 (Springel, 2005), in which particle trajectories are integrated with individual timesteps and synchronised with the largest timestep. As we allow the block time step ${ }^{3}$ to vary with time, we work with the so called KDK approach (KickDrift-Kick) in which velocties are updated in two half steps whereas position is updated in a full step. It can be shown that with a variable time step, KDK performs better than DKD (Drift-Kick-Drift) (see the GADGET-2 paper (Springel, 2005) for details.). In our implementation of the hierarchy of time steps, the smaller time steps differ by an integer power $(n)$ of 2 from the largest, block time step. An array is then used to store the value $n$ which determines the timestep of the particle. The code drifts all the particles with the smallest timestep to the next time, where a force computation is done for particles that require an updation of velocity (Kick). We have tested the robustness of the hierarchical KDK integrator by succesfully integrating the 3 -body problem discussed by Szebehely \& Peters, (Szebehely \& Peters, 1967).

Solving the equation of motion with a hierarchy of time steps can be combined with the group scheme. Since tree construction takes a small fraction of the total time, a new tree can be constructed whenever particles require an updation of velocity. The groups that contain such particles can then be identified and particles within each group can be reordered into two disjoint sets: ones that need an updation of velocity and others that don't. Force is computed only for particles in the first set. Since each group represents a very small fraction of the total number of particles, the overhead of reordering particles is negligible.

Table 2 lists the time taken for a complete simulation run for the unoptimised TreePM, TreePM with hierarcical time steps, TreePM with the group scheme, and finally the TreePM with the group scheme as well as the hierarchical time steps and their speedup with respect to the base TreePM. The model used for this comparison is a power law model with $n=-1.0, N_{\text {box }}^{3}=N=64^{3}$. We used $r_{s}=1.0, r_{\text {cut }}=5.2 r_{s}$, $\theta_{c}=0.5$ and $\epsilon=0.2$ in all the runs. Here $\epsilon$ is the softening length. We used $c_{\max }=4.0$ and $n_{p_{\max }}=1024$ for the modified TreePM.

We note that the hierarchical integrator gives a speedup of better than a factor 2, irrespective of whether the scheme of groups is used or not. The speedup is larger if the softening $\epsilon$ is smaller, as the number of levels in the hierarchy increases with decreasing $\epsilon$. The scheme of groups on the other hand gives a speedup of 4 or better for small simulations, and a much larger speedup for bigger simulations. This speedup has little dependence on the TreePM parameters, i.e. $\theta_{c}, r_{s}, r_{c u t}$. The combination of two optimisations gives us a speedup of 10 or more even for small simulations.

\section{DISCUSSION}

The scheme of groups when combined with a hierarchical integrator for the equation of motion guarantees a speedup of better than 10 for any $N$-body code which uses tree structures for computing forces. From

\footnotetext{
3 Same as the largest time step.
} 
the algorithmic point of view one does not expect a much larger speedup for larger $N$. However, as seen in Figure 2, the scheme also allows us to make better use of the cache on CPUs and the effective speedup can be even more impressive. We have demonstrated that memory overhead is negligible, and as was observed in (Barnes, 1990) this optimisation just takes around 200 extra lines of code. A welcome feature is more accurate force computation than the code without this modification. This modification in principle introduces two additional parameters $\left(c_{\max }, n_{p_{\max }}\right)$, but these are not independent and we have found that $c_{\max } \sim r_{\text {cut }}$ and $n_{p_{\max }} \geq 10^{3}$ are good choices across a range of simulation sizes.

Our analysis of the optimisation has been restricted to fixed resolution simulations. In case of zoom-in simulations the range of time scales is much larger and a more complex approach for combining the group scheme with the hierarchy of time steps may be required. Relative efficacy of the two optimisations may be very different in such a case when compared with the example studied in the previous section.

In summary, we would like to point out that the scheme of groups leads to a significant optimisation of the TreePM method. The amount of CPU time saved is significant even for small simulations, but the cache optimisation aspect leads to even more significant gains for large simulations. We have shown in this paper, that it is possible to incorporate the scheme in a simple manner in any tree based code. The overall gain is very impressive as we are able to combine this with the use of a hierarchy of time steps. The possibility of combining the two optimisations has been explored in this work for the first time.

Acknowledgements Numerical experiments for this study were carried out at cluster computing facility in the Harish-Chandra Research Institute (http://cluster.hri.res.in). This research has made use of NASA's Astrophysics Data System. The authors would like to thank Hugh Couchman, Jun Makino and Volker Springel for useful comments.

\section{References}

Bagla J. S., 2005, CSci, 88, 1088

Bagla J. S., 2002, JApA, 23, 185

Bagla J. S., Padmanabhan T., 1997, Prama, 49, 161

Bagla J. S., Padmanabhan T., 1994, MNRAS, 266, 227

Bagla J. S., Ray S., 2003, NewA, 8, 665

Barnes J., Hut P., 1986, Natur, 324, 446

Barnes J. E., 1990, JCoPh, 87, 161

Bernardeau F., Colombi S., Gaztañaga E., Scoccimarro R., 2002, PhR, 367, 1

Bertschinger E., 1998, ARA\&A, 36, 599

Bode P., Ostriker J. P., 2003, ApJS, 145, 1

Bode P., Ostriker J. P., Xu G., 2000, ApJS, 128, 561

Bouchet F. R., Adam J.-C., Pellat R., 1985, A\&A, 144, 413

Bouchet F. R., Kandrup H. E., 1985, ApJ, 299, 1

Bouchet F. R., Hernquist L., 1988, ApJS, 68, 521

Brainerd T. G., Scherrer R. J., Villumsen J. V., 1993, ApJ, 418, 570

Brieu P. P., Evrard A. E., 2000, NewA, 5, 163

Brieu P. P., Summers F. J., Ostriker J. P., 1995, ApJ, 453, 566

Couchman H. M. P., 1991, ApJ, 368, L23

Davis M., Peebles P. J. E., 1977, ApJS, 34, 425

Dehnen W., 2002, JCoPh, 179, 27

Dehnen W., 2000, ApJ, 536, L39

Dubinski J., 1996, NewA, 1, 133

Dubinski J., Kim J., Park C., Humble R., 2004, NewA, 9, 111

Ebisuzaki T., Makino J., Fukushige T., Taiji M., Sugimoto D., Ito T., Okumura S. K., 1993, PASJ, 45, 269

Efstathiou G., Davis M., White S. D. M., Frenk C. S., 1985, ApJS, 57, 241

Efstathiou G., Frenk C. S., White S. D. M., Davis M., 1988, MNRAS, 235, 715

Greengard L., Rokhlin V., 1987, JCoPh, 73, 325

Gurbatov S. N., Saichev A. I., Shandarin S. F., 1989, MNRAS, 236, 385

Hamilton A. J. S., Kumar P., Lu E., Matthews A., 1991, ApJ, 374, L1 
Hernquist L., 1990, JCoPh, 87, 137

Hernquist L., 1987, ApJS, 64, 715

Hernquist L., Bouchet F. R., Suto Y., 1991, ApJS, 75, 231

Hockney R. W., Eastwood J. W., 1988, Computer Simulation using Particles, McGraw-Hill

Hui L., Bertschinger E., 1996, ApJ, 471, 1

Jain B., Mo H. J., White S. D. M., 1995, MNRAS, 276, L25

Jernigan J. G., Porter D. H., 1989, ApJS, 71, 871

Kanekar N., 2000, ApJ, 531, 17

Kawai A., Makino J., 2001, ApJ, 550, L143

Klypin A. A., Shandarin S. F., 1983, MNRAS, 204, 891

Knebe A., Green A., Binney J., 2001, MNRAS, 325, 845

Kravtsov A. V., Klypin A. A., Khokhlov A. M., 1997, ApJS, 111, 73

Ma C.-P., 1998, ApJ, 508, L5

Macfarland T., Couchman H. M. P., Pearce F. R., Pichlmeier J., 1998, NewA, 3, 687

Makino J., 2004, PASJ, 56, 521

Makino J., 2002, NewA, 7, 373

Makino J., 1991, PASJ, 43, 621

Makino J., 1990, JCoPh, 87, 148

Makino J., Fukushige T., Koga M., Namura K., 2003, PASJ, 55, 1163

Matarrese S., Lucchin F., Moscardini L., Saez D., 1992, MNRAS, 259, 437

Merz H., Pen U.-L., Trac H., 2005, NewA, 10, 393

Miller R. H., 1983, ApJ, 270, 390

Nityananda R., Padmanabhan T., 1994, MNRAS, 271, 976

Padmanabhan T., 2002, Theoretical Astrophysics, Volume III: Galaxies and Cosmology. Cambridge University Press.

Padmanabhan T., 1996, MNRAS, 278, L29

Padmanabhan T., Cen R., Ostriker J. P., Summers F. J., 1996, ApJ, 466, 604

Peacock J. A., Dodds S. J., 1996, MNRAS, 280, L19

Peacock J. A., Dodds S. J., 1994, MNRAS, 267, 1020

Peacock J. A., 1999, Cosmological Physics, Cambridge University Press

Peebles P. J. E., 1980, The Large-Scale Structure of the Universe, Princeton University Press

Peebles P. J. E., 1974, A\&A, 32, 391

Percival W. J., et al., 2007, ApJ, 657, 645

Ray S., Bagla J. S., 2004, astro, arXiv:astro-ph/0405220

Sahni V., Coles P., 1995, PhR, 262, 1

Salmon J. K., Warren M. S., 1994, JCoPh, 111, 136

Smith R. E., et al., 2003, MNRAS, 341, 1311

Spergel D. N., et al., 2007, ApJS, 170, 377

Springel V., Yoshida N., White S. D. M., 2001, NewA, 6, 79

Springel V., 2005, MNRAS, 364, 1105

Springel V., et al., 2005, Natur, 435, 629

Suisalu I., Saar E., 1995, MNRAS, 274, 287

Szebehely V., Peters C. F., 1967, AJ, 72, 1187

Thacker R. J., Couchman H. M. P., 2006, CoPhC, 174, 540

Theuns T., 1994, CoPhC, 78, 238

Wadsley J. W., Stadel J., Quinn T., 2004, NewA, 9, 137

Xu G., 1995, ApJS, 98, 355

Yoshikawa K., Fukushige T., 2005, PASJ, 57, 849

Zel'Dovich Y. B., 1970, A\&A, 5, 84 